\title{
Reconstitution of Respiratory Complex I on a Biomimetic Membrane Supported on Gold Electrodes
}

\author{
Oscar Gutiérrez-Sanz, ${ }^{\mathrm{a}}$ David Olea, ${ }^{\mathrm{a}}$ Marcos Pita, ${ }^{\mathrm{a}}$ Ana P. Batista, ${ }^{\mathrm{b}}$, Alvaro Alonso, ${ }^{\mathrm{a}}$ Manuela M. Pereira, ${ }^{\mathrm{b}}$ \\ Marisela Vélez, ${ }^{\mathrm{a}, \mathrm{c}}$ Antonio L. De Lacey ${ }^{\mathrm{a} *}$ \\ ${ }^{a}$ Instituto de Catalisis y Petroleoquímica, CSIC, c/ Marie Curie 2, L10, 28049 Madrid, Spain. Fax: +34 \\ 91585 4760; Tel: +34 91585 4813; E-mail: alopez@icp.csic.es \\ ${ }^{b}$ Instituto de Tecnologia Química e Biológica, Universidade Nova de Lisboa, Apartado 127, 2781-901 \\ Oeiras, Portugal. \\ ${ }^{c}$ Instituto Madrileño de Estudios Avanzados en Nanociencia (IMDEA-Nanociencia), Facultad de \\ Ciencias, C-IX-3 ${ }^{a}$ Cantoblanco 28049, Madrid, Spain. \\ KEYWORDS: complex I, NADH:ubiquinone oxidoreductase, biomimetic membrane, bioelectrochemistry, \\ thiol SAM
}

\begin{abstract}
For the first time respiratory complex I has been reconstituted on an electrode preserving its structure and activity. Respiratory complex I is a membrane-bound enzyme that has an essential function in cellular energy production. It couples NADH:quinone oxidoreduction to translocation of ions across the cellular (in prokaryotes) or mitochondrial membranes. Therefore, complex I contributes to the establishment and maintenance of the transmembrane difference of electrochemical potential required for ATP synthesis, transport and motility. Our new strategy has been applied for reconstituting the bacterial complex I from Rhodothermus marinus onto a biomimetic membrane supported on gold electrodes modified with a thiol self-assembled monolayer (SAM). Atomic force microscopy and faradaic impedance measurements give evidence of the biomimetic construction, whereas electrochemical
\end{abstract}


measurements show its functionality. Both electron transfer and proton translocation by respiratory complex I were monitored, simulating in vivo conditions.

\section{Introduction}

Life depends on continuous flow of energy and on mechanisms that control this energy flow. Respiratory complex I (E.C.1.6.5.3) is an energy transducing enzyme present in the three domains of life. This enzyme catalyzes the oxidation of NADH and the reduction of quinone coupled to ion translocation across the membrane. In this way, it contributes to the establishment of the transmembrane difference of electrochemical potential which is used for the synthesis of ATP, solute transport and motility. Deficiencies in this complex have been shown to be implicated in several pathologies, namely neurodegenerative diseases such as Leber's hereditary optic neuropathy, Parkinson and Dystonia disorders. $^{1}$

In mitochondria, the enzyme is composed of 45 subunits with a total molecular mass of $1 \mathrm{MDa}$, whereas the bacterial complex I, considered to comprise the minimal functional unit, has in general 14 subunits (named Nqo1-14 or NuoA-N) with a total mass of $550 \mathrm{kDa}$. The subunits are arranged in an Lshaped structure, consisting of a peripheral and a membrane arms as shown in Fig. $1 .^{2-5}$ The peripheral part contains the prosthetic groups (iron-sulfur centers and FMN), ${ }^{2}$ while the membrane part is, most likely, involved in quinone reduction and charge translocation. ${ }^{5}$ The nature of the ion(s) translocated by this enzyme is still a highly discussed issue, being $\mathrm{H}^{+}$and $\mathrm{Na}^{+}$possible candidates. It is generally accepted that $\mathrm{H}^{+}$is the coupling ion in complex $\mathrm{I},{ }^{6,7}$ however in the case of some bacterial complexes $\mathrm{I} \mathrm{Na}^{+}$has been proposed to have that role. ${ }^{8,9}$ It was also shown that some bacterial complexes I are capable of $\mathrm{H}^{+}$and $\mathrm{Na}^{+}$ translocation, but in opposite directions, with $\mathrm{H}^{+}$being the coupling ion. ${ }^{10-12}$

The study of this enzyme is currently a hot topic in bioenergetics, stimulated by recently structural and functional data ${ }^{2,5}$. Still, the mechanisms of quinone reduction, ion translocation and their coupling are unknown. To our knowledge, the study of both redox and ion translocation activities in the same experimental setup was not full achieved yet. Different strategies and techniques have been employed to 
study NADH oxidation, mostly working with soluble subcomplexes or partially solubilized proteins for using electrochemical and spectroscopic techniques. ${ }^{13-16}$ In order to study proton translocation, the complex I has to be inserted in a lipid bilayer that provides the enzyme with the appropriate lipid environment and separates two compartments needed to maintain the proton gradient. Protein orientation and compartmentalization can be achieved by working with intact lipid vesicles obtained from cell fractions, ${ }^{10,11,17,18}$ or by reconstituting the isolated protein in liposomes. ${ }^{12}$ Such reconstitution protocols are not appropriate for using dynamic electrochemical techniques, which have proven to be powerful tools to study thermodynamic and kinetic properties of proteins, but require their connection to the electrode surface. ${ }^{19}$

The immobilization of purified redox membrane enzymes on surfaces modified with model membranes opens the possibility of studying them by a combination of methodologies that provide information about both the catalytic process and the associated structural rearrangements. ${ }^{20}$ This information is very difficult to achieve in lipid vesicle solutions obtained from cell membrane fragments. Several strategies have been developed for building biomimetic membranes on conductive supports, ${ }^{21}$ which allow using electrochemical, ${ }^{22-24}$ spectroscopic $^{25-28}$ and atomic force microscopy (AFM) (23,26 $^{23}$ techniques for studying immobilized membrane proteins. However, to our knowledge these strategies have not been employed for studying respiratory complex I. Reconstitution of the respiratory complex I, in its native form on supported biomimetic membranes should allow performing many fundamental studies about its function in cellular energy production, and may also lead to the development of devices for mitochondrial toxicity screening. ${ }^{29}$

In this study we have developed a strategy for reconstituting the bacterial complex I from Rhodothermus marinus, keeping its structural and functional properties, onto a biomimetic membrane supported on gold electrodes modified with a thiol self-assembled monolayer (SAM), as shown in Fig. 1. We show that this biomimetic construction allows studying electrochemically both the electron transfer and the proton translocation by complex I. 


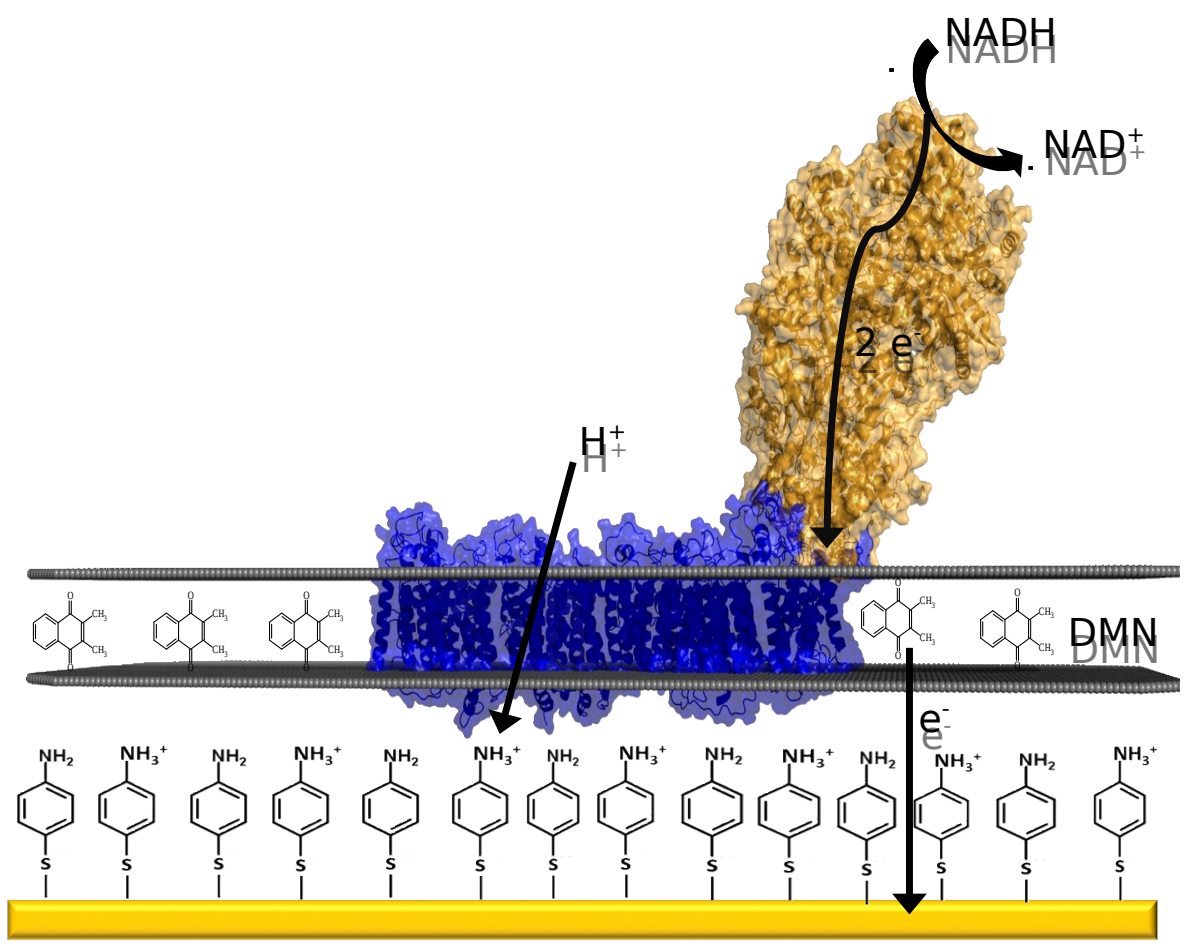

Figure 1. Schematic representation of complex I reconstituted in a phospholipid bilayer containing DMN over a gold electrode modified with 4-ATP. Complex I oxidizes NADH transferring the electrons to the DMN present in the bilayer, which acts as redox mediator at the electrode. The enzymatic redox process is coupled to proton translocation across the biomimetic membrane.

\section{Materials and methods}

Bacterial growth and protein purification. Rhodothermus marinus growth, membrane preparation, and membrane protein solubilization were done as described previously, ${ }^{30}$ except that the growth medium contained $100 \mathrm{mM}$ glutamate. Respiratory NADH:menaquinone oxidoreductase (complex I) was purified according to an established procedure, ${ }^{31}$ optimized by introducing a further chromatographic step with a Mono-Q column (GE-Healthcare). Briefly, the sample was submitted to two successive High Performance Q-Sepharose columns, using as buffer $20 \mathrm{mM}$ Tris-HCl (pH 8.0), $1 \mathrm{mM}$ phenylmethanesulfonyl fluoride (PMSF, Roth), and 0.1\% n-dodecyl-b-D-maltoside (DDM, Glycon 
Biochemicals $\mathrm{GmbH}$ ), and was eluted in a linear gradient, 0 to $1 \mathrm{M} \mathrm{NaCl}$. The fraction containing complex-I was then applied to a gel filtration S200 column, eluted with $20 \mathrm{mM}$ Tris-HCl (pH 8.0), $1 \mathrm{mM}$ PMSF, 0.1\% DDM, and $150 \mathrm{mM} \mathrm{NaCl}$, and finally applied on a Mono Q column using 20 mM Tris-HCl (pH 8.0), 1 mM PMSF, and 0.1\% DDM as buffer. The complex I was eluted in a linear gradient, 0 to $1 \mathrm{M} \mathrm{NaCl}$.

Gold surface preparation. Au wire $(0.25 \mathrm{~mm}$ radius $)$ and Au-coated substrates $\left(1 \mathrm{x} 1 \mathrm{~cm}^{2}\right)$ were purchased from Goodfellow Cambridge Limited and Metallhandel Schroer GMBH respectively. The substrates for AFM experiments had an Au layer of $200 \mathrm{~nm}$ thickness Au over 1-4 nm of Cr prepared on borosilicate glass. The substrates were cleaned with piranha solution $\left(3: 1 \mathrm{H}_{2} \mathrm{SO}_{4} \quad 98 \%: \mathrm{H}_{2} \mathrm{O}_{2} \quad 30 \%\right)$ (Caution! Piranha solution is especially dangerous and corrosive and may explode if contained in a closed vessel. It should be handled with special care) and rinsed extensively with Milli Q water. The substrates were then annealed to an orange glow for a few seconds in a propane flame; this operation was repeated five times. This treatment is known to produce Au (111) terraces of a few micrometers radius with atomically flat surfaces separated by deep boundaries, suitable for AFM characterization (Fig. S1 of Supporting Information). Au wires were treated as the Au-coated substrates.

Preparation of liposomes. $200 \mu \mathrm{L}$ of $10 \mathrm{mg} / \mathrm{mL}$ egg phosphatidylcholine (PC, Avanti) in choloroform were mixed with $180 \mu \mathrm{L}$ of $1 \mathrm{mg} / \mathrm{mL}$ egg phosphatidic acid (PA, Avanti) in the same solvent and with $35 \mu \mathrm{L}$ of $1.5 \mathrm{mg} / \mathrm{mL}$ 2,3-dimethyl-1,4-naphthoquinone (DMN, synthesized as described) ${ }^{32}$ in ethanol. The solution was shaken 10 minutes and then evaporated under $\mathrm{N}_{2}$ flow. Phosphate buffer $0.1 \mathrm{M}$, $\mathrm{pH} 5.5$ was then added to form a $2 \mathrm{mg} / \mathrm{mL}$ suspension of phospholipids that was immersed in an ultrasound bath for 15 min. The suspension was then extruded with an Avanti extruder equipped with a porous membrane ( $1 \mu \mathrm{m}$ pore diameter). This procedure leads to the formation of a quasi-monodisperse suspension of unilamelar vesicles.

Preparation of the proteoliposomes. Two types of liposomes were used; 9 PC:1 PA in weight liposomes with and without DMN. In both cases the complex I was inserted in the same way; $10 \mu \mathrm{L}$ of 6,2 $\mathrm{mg} / \mathrm{mL}$ complex I were added to $500 \mu \mathrm{L}$ of the liposome suspension and stirred with Vortex at $4^{\circ} \mathrm{C}$ during 
150 min, adding 15 mg of CALBIOSORB adsorbent biobeads (Calbiochem) every 45 min of stirring. The biobeads were added to remove the detergent from the complex I sample, thus facilitating its reconstitution in the liposomes. The biobeads were washed with $0.1 \mathrm{M}$ phosphate buffer, $\mathrm{pH} 5.5$ before use.

Modification of the gold surfaces. First, the clean Au (111) surfaces were modified with a 4aminothiophenol (4-ATP, Sigma) monolayer as reported. ${ }^{23}$ The 4-ATP/Au was incubated in liposomes or proteoliposomes suspension overnight at $4^{\circ} \mathrm{C}$ in the presence of biobeads.

Electrochemical measurements. The measurements were run in a three-electrode glass cell with an $\mathrm{Ag} / \mathrm{AgCl}$ (3 $\mathrm{M} \mathrm{NaCl}$ ) reference electrode from BAS or a saturated calomel reference electrode from Radiometer. The working electrodes had a geometric area of $0.065 \mathrm{~cm}^{2}$. All the results are shown vs. normal hydrogen electrode, NHE. A platinum wire from Goodfellow Cambridge Limited was used as a counter electrode. All the measurements were perfomed under $\mathrm{N}_{2}$ atmosphere at $25^{\circ} \mathrm{C}$ with an Autolab PGSTAT30 potentiostat/galvanostat or a $\mu$-Autolab III electrochemical analyzer controlled by GPES 4.9 software (Eco Chemie). The Faradaic impedance spectra were recorded using FRA 4.9 software (Eco Chemie) while applying $+0.44 \mathrm{~V}$ (vs. NHE) bias potential and using $10 \mathrm{mV}$ alternative voltage in the frequency range $10 \mathrm{kHz}-100 \mathrm{mHz}$. The experimental impedance spectra were fitted using electronic equivalent circuits (Randles and Ershler model) ${ }^{33}$ in order to derive the electron-transfer resistance, $\mathrm{R}_{\mathrm{et}}$, values. For this purpose, the same FRA software was used. A mixture of $2.5 \mathrm{mM} \mathrm{K} \mathrm{K}_{4}\left[\mathrm{Fe}(\mathrm{CN})_{6}\right]$ and $2.5 \mathrm{mM}$ $\mathrm{K}_{3}\left[\mathrm{Fe}(\mathrm{CN})_{6}\right]$ was used as electrochemical probe, dissolved in $100 \mathrm{mM}$ phosphate buffer $\mathrm{pH}$ 7. The measurements were carried out at room temperature $\left(23 \pm 2{ }^{\circ} \mathrm{C}\right)$

Atomic Force Microscopy (AFM) measurements. An Agilent Technologies 5500 microscope was used for AFM imaging. Measurements were always made under liquid conditions in $0.1 \mathrm{M}$ phosphate buffer, pH 7 at room temperature using Olympus rectangular silicon nitride cantilevers (RC800PSA, $200 \times$ $20 \mu \mathrm{m}^{2}$ ) with a spring constant of $0.05 \mathrm{~N} / \mathrm{m}$, an estimated tip radius of $20 \mathrm{~nm}$, and a resonance frequency 
in the liquid cell of approximately $27 \mathrm{kHz}$. Scanning rates were kept close to $1 \mathrm{~Hz}$. All images contain 512 pixels x 512 pixels and were first-order flattened using Picoimage software from Agilent.

\section{Results}


AFM study of reconstituted complex I. The structural characterization of the modified gold surfaces was carried out using AFM microscopy. The flat gold surfaces were treated according to the same protocol established to modify the gold wires used for the electrochemical measurements. Fig. 2a,b show images of the gold surface modified with a 4-ATP SAM after overnight incubation in a complex I proteoliposomes suspension at $4^{\circ} \mathrm{C}$. Fig. S1a,b in Supporting Information show, for comparison, an AFM image and z-axis profile respectively of a bare annealed gold surface. Evidence that the gold surface was covered by a soft lipid biomembrane comes from the indentation curve shown in Fig. 2c. It indicates that the AFM tip, upon approach to the surface, penetrated through a soft material, approximately $5 \mathrm{~nm}$ in depth, before reaching the hard underlying gold substrate. This thickness agrees with that expected for a phospholipid bilayer lying on top of the 4-ATP SAM-modified surface. ${ }^{23}$ Further experimental evidence of the phospholipid bilayer formation was obtained from the image of a modified surface in which a region of the biomimetic membrane was removed by scratching with the tip (Fig. S1c,d in Supporting Information).
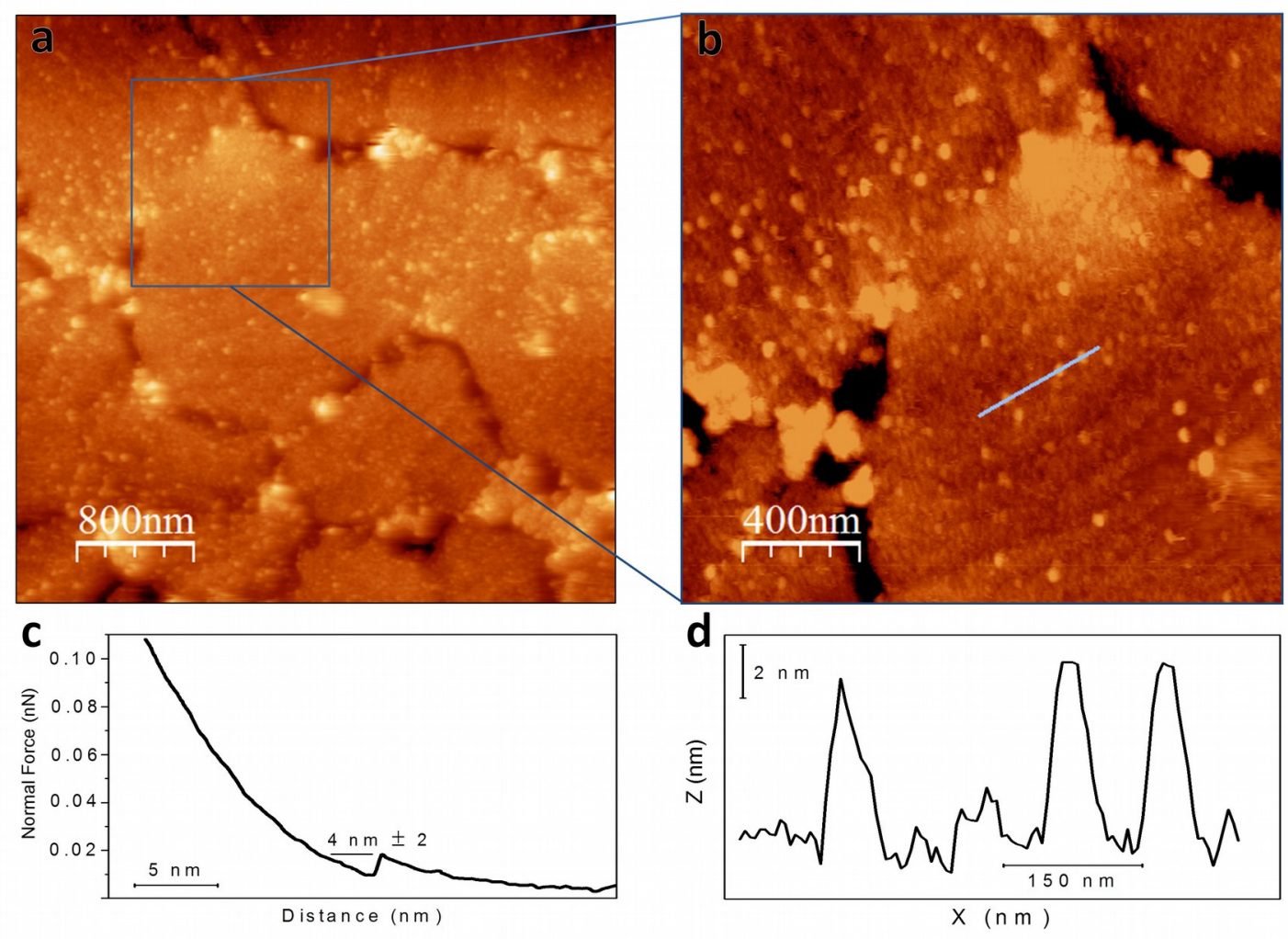
Figure 2. AFM images of reconstituted complex I: (a) and (b) tapping-mode AFM topography of a 4ATP-modied gold plate to which respiratory complex I has been immobilized in presence of phospholipids and CALBIOSORB adsorbent; (c) force-distance (z axis) curve acquired over a flat gold area; (d) z-axis profile across the line drawn in (b).

The modified surface presented protrusions whose height of 6-8 nm, as seen in Fig. 2d, correlate well with values expected for the hydrophilic arm of complex I extending outside the membrane (Fig. 1). The lateral dimensions observed are ultimately determined by the tip diameter; therefore we can only reliably estimate that the height of the proteins is indeed within the range expected for the soluble part sticking out of the membrane. The density of the protrusions observed on the flat regions of the gold terraces falls within the expected $4-10 \%$ surface area covered by protein, considering that the lipid to protein molar ratio used to prepare the samples was 7000:1.

Impedance characterization of the biomimetic construction over Au electrodes. The reconstitution of the membrane-bound enzyme on Au wire electrodes was monitored step-by-step using faradaic impedance spectroscopy. The impedance spectra were recorded for each of the following steps of the electrode modification: bare $\mathrm{Au}, \mathrm{Au}$ modified with 4-ATP (Au/4-ATP), Au modified with the phospholipid bilayer (Au/4-ATP/PhL) and the complete construction with reconstituted complex I (Au/4$\mathrm{ATP} / \mathrm{PhL}+\mathrm{C}-\mathrm{I})$.

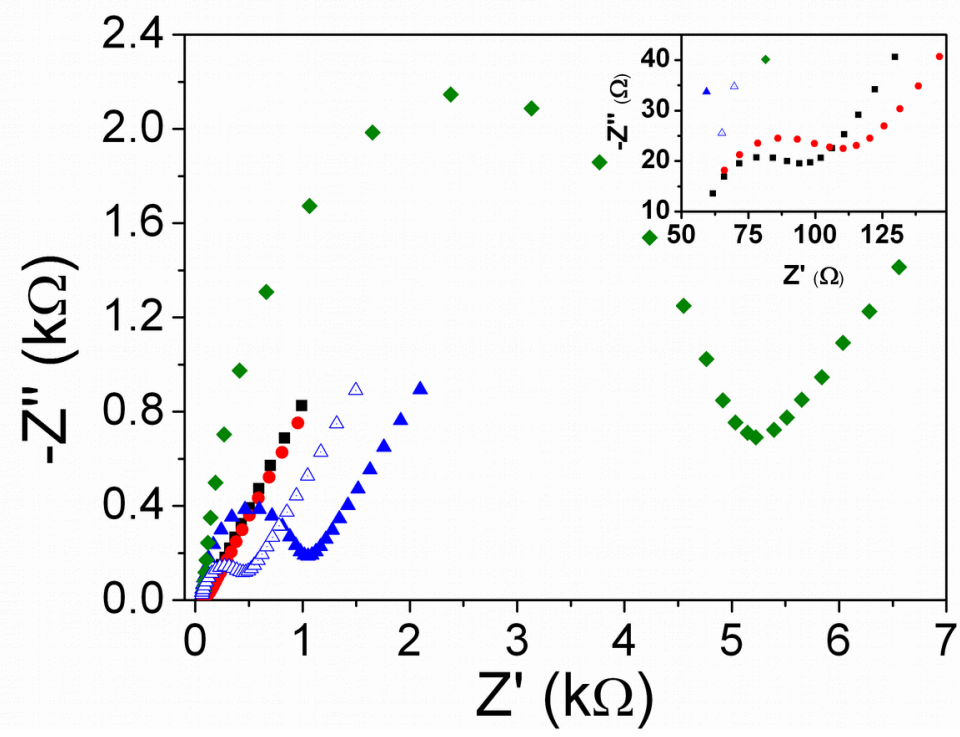


Figure 3. Faradaic impedance spectra obtained in the presence of $2.5 \mathrm{mM} \mathrm{K}{ }_{3} \mathrm{Fe}(\mathrm{CN})_{6}$ and $2.5 \mathrm{mM}$ $\mathrm{K}_{4} \mathrm{Fe}(\mathrm{CN})_{6}$ for bare Au electrode (squares), Au/4-ATP electrode (circles), Au/4-ATP/PhL electrode formed by $3 \mathrm{~h}$ room temperature incubation (void triangles), Au/4-ATP/PhL electrode formed by overnight $4{ }^{\circ} \mathrm{C}$ incubation (solid triangles) and $\mathrm{Au} / 4-\mathrm{ATP} / \mathrm{PhL}+\mathrm{C}-\mathrm{I}$ electrode (diamonds). The background solution was composed of 0.1 M phosphate buffer, pH 7.0.

The impedance spectra were represented as Nyquist plots (Fig. 3 and the fitted parameters are given in Table S1 of the Supporting Information. The impedance spectrum measured on the bare Au electrode (Fig. 3, squares) showed a typical response dominated by the Warburg process. ${ }^{34}$ However, from the highest frequency data a charge resistance of $50 \Omega$ can be estimated (inset of Fig. 3, squares). Taking into account the geometric electrode area, this corresponds to ca. $3 \Omega \times \mathrm{cm}^{2}$. The impedance spectrum measured for the Au/4-ATP electrode also showed an electrical resistance $\left(\mathrm{R}_{\mathrm{et}}\right)$ lower than $5 \Omega \times \mathrm{cm}^{2}$, indicating that the assembled monolayer is not insulating the surface (Fig. 3, circles). The bilayer formation on Au/4-ATP electrodes was studied by incubation of the electrode in the phospholipids suspension under two different conditions: (i) at RT during 3 hours and (ii) overnight at $4^{\circ} \mathrm{C}$. An impedance spectrum of each $\mathrm{Au} / 4-\mathrm{ATP} / \mathrm{PhL}$ electrode was recorded. The impedance spectrum measured for the $\mathrm{Au} / 4-\mathrm{ATP} / \mathrm{PhL}$ (3 hours) electrode showed a $\mathrm{R}_{\mathrm{et}}$ of ca. $33 \Omega \times \mathrm{cm}^{2}$, which is 7 times higher than the $\mathrm{R}_{\mathrm{et}}$ measured for the Au/4-ATP electrode (Fig. 3, void triangles). The impedance spectrum measured for the overnight Au/4-ATP/PhL electrode showed a $\mathrm{R}_{\mathrm{et}}$ of ca. $72 \Omega \times \mathrm{cm}^{2}$, which is a 15 -fold increase on the previous step (Fig. 3, solid triangles). These results evidence the presence of an additional charge-transfer barrier on the $\mathrm{Au} / 4-\mathrm{ATP} / \mathrm{PhL}$ electrode, in agreement with the formation of a phospholipid bilayer. Moreover, the difference in the $\mathrm{R}_{\mathrm{et}}$ measured for the fast-formed bilayer and the slow-formed one suggests a more densely packed and less prone to defects bilayer in the latter case. Thus, the overnight bilayer formation method was used in the further experiments. The last stage of the reconstitution of complex I in the biomimetic membrane, $\mathrm{Au} / 4-\mathrm{ATP} / \mathrm{PhL}+\mathrm{C}-\mathrm{I}$, was also measured. The impedance spectrum of $\mathrm{Au} / 4$ - 
$\mathrm{ATP} / \mathrm{PhL}+\mathrm{C}$-I gave a remarkably higher $\mathrm{R}_{\mathrm{et}}$ of ca. $0.360 \mathrm{k} \Omega \times \mathrm{cm}^{2}$, which is a 5-fold increase over the $\mathrm{R}_{\mathrm{et}}$ measured for the overnight $\mathrm{Au} / 4-\mathrm{ATP} / \mathrm{PhL}$ (Fig. 3, diamonds).

\section{Electrocatalytic oxidation of NADH by the reconstituted complex $I$ in the presence of}

quinone. The electrocatalytic properties of the reconstituted complex I on the gold electrodes modified with the biomimetic membrane were studied by cyclic voltammetry. The quinone DMN was incorporated in the phospholipid bilayer as electron acceptor of the enzyme and redox mediator with the electrode (Fig. 1). Several control experiments were done with modified electrodes in absence of either the phospholipid bilayer, the complex I or the redox mediator.
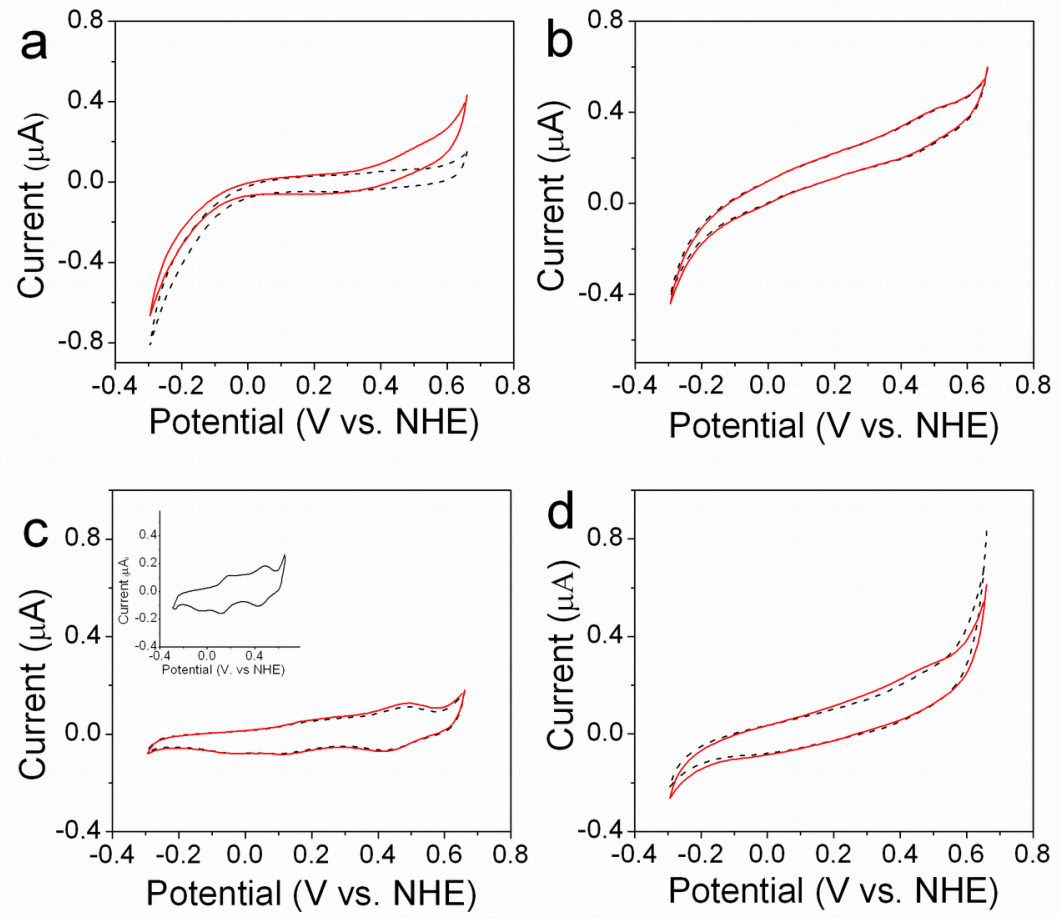

Figure 4. CVs of control electrodes performed at $50 \mathrm{mV} / \mathrm{s}$ in $0.1 \mathrm{M}$ phosphate buffer, $\mathrm{pH} 7.0$ in the absence (dashed line) or in the presence of $60 \mu \mathrm{M}$ NADH (solid line): (a) Au/4-ATP electrode; (b) Au/4ATP/PhL electrode; (c) Au/4-ATP/PhL+DMN electrode, the inset graph shows the result at $100 \mathrm{mV} / \mathrm{s}$; (d) $\mathrm{Au} / 4-\mathrm{ATP} / \mathrm{PhL}+\mathrm{C}-\mathrm{I}$. 
First of all, cyclic voltammograms (CVs) for an Au/4-ATP electrode were measured in either the presence or absence of NADH. Fig. 4a shows the direct oxidation of NADH on the electrode, which onsets at $\mathrm{E}=+300 \mathrm{mV}$ potential. Such oxidation is known to be an irreversible process, ${ }^{35}$ which is confirmed by the absence of an electrochemical reduction process for $\mathrm{NAD}^{+}$. The cathodic current observed at negative potentials is also present in the CV recorded in absence of NADH, and may be attributed to non-scavenged oxygen at the electrode boundary. The formation of a phospholipid bilayer on top of the Au/4-ATP electrode avoided the electrochemical oxidation of NADH (Fig. 4b). This result shows that the phospholipid bilayer is acting as a barrier to NADH diffusion towards the electrode surface.

Fig. 4c shows the $\mathrm{CVs}$ measured at $50 \mathrm{mV} / \mathrm{s}$ with a $\mathrm{Au} / 4-\mathrm{ATP} / \mathrm{PhL}+\mathrm{DMN}$ electrode. The electrochemistry response of the embedded naphthoquinone is a quite complex process that provides the CVs with several reduction and oxidation peaks. This electrochemical behavior is typical for quinone derivatives at neutral $\mathrm{pH} .{ }^{24,36-39}$ A CV recorded at $100 \mathrm{mV} / \mathrm{s}$ scan rate allows a better resolution of the DMN redox waves (Fig. 4c inset). The redox pair centered at $+450 \mathrm{mV}$ can also be found in the CV recorded for an $\mathrm{Au} / 4-\mathrm{ATP} / \mathrm{PhL}$ electrode in the absence of DMN (Fig. 4b), thus it is not assigned to the naphthoquinone derivative. When the electrode was scanned several times between -0.1 and $+0.7 \mathrm{~V}$ vs. $\mathrm{Ag} / \mathrm{AgCl}$ the peak intensities of both oxidation and reduction waves centered at $+450 \mathrm{mV}$ increased (Fig. S2a in Supporting Information). Therefore, this quasi-reversible redox process is attributed to surfaceconfined aniline dimers formed by oxidation of part of the 4-ATP monolayer molecules, as reported by Raj et al.. ${ }^{40}$ By contrast the redox signals assigned to DMN (reduction peaks at $+120 \mathrm{mV}$ and $-65 \mathrm{mV}$, oxidation peak at $+180 \mathrm{mV}$ ) decreased with the number of cycles recorded (Fig. S2b of Supporting Information). This suggests that the reduced naphthoquinone maybe leaking out of the phospholipid bilayer due to its higher hydrophilicity. Addition of NADH to the solution only resulted in an insignificant increase of the oxidative peak current at $+480 \mathrm{mV}$ (Fig. 4c). Thus, the bilayer dramatically hindered the direct oxidation of $\mathrm{NADH}$, whereas the presence of embedded DMN hardly allowed mediated electrochemical oxidation of NADH. An additional control experiment was done with an Au/4ATP/PhL+C-I electrode, but excluding DMN from the experiment (Fig. 4d). Again, the increase of 
oxidation currents was negligible when $\mathrm{NADH}$ was added to the solution, indicating that direct electrocatalytic oxidation of NADH by the reconstituted complex I was negligible.

Fig. 5a,b shows the CVs measured for a $\mathrm{Au} / 4-\mathrm{ATP} / \mathrm{PhL}+\mathrm{C}-\mathrm{I}+\mathrm{DMN}$ electrode that included the naphthoquinone in the phospholipid bilayer as electron acceptor from complex I. An increase of the anodic currents was observed by $\mathrm{CV}$ when $\mathrm{NADH}$ was added to the solution, which is explained by an electrocatalytic procces due to NADH oxidation by the reconstituted complex I and redox-mediated by DMN. The measured electrocatalytic enhancement, starting at $-0.1 \mathrm{~V}$, was greater when the CV was recorded at $5 \mathrm{mV} / \mathrm{s}$. This higher ratio of the catalytic current relative to the non-catalytic current at slower scan rates indicates that the overall electrocatalytic process is slow.
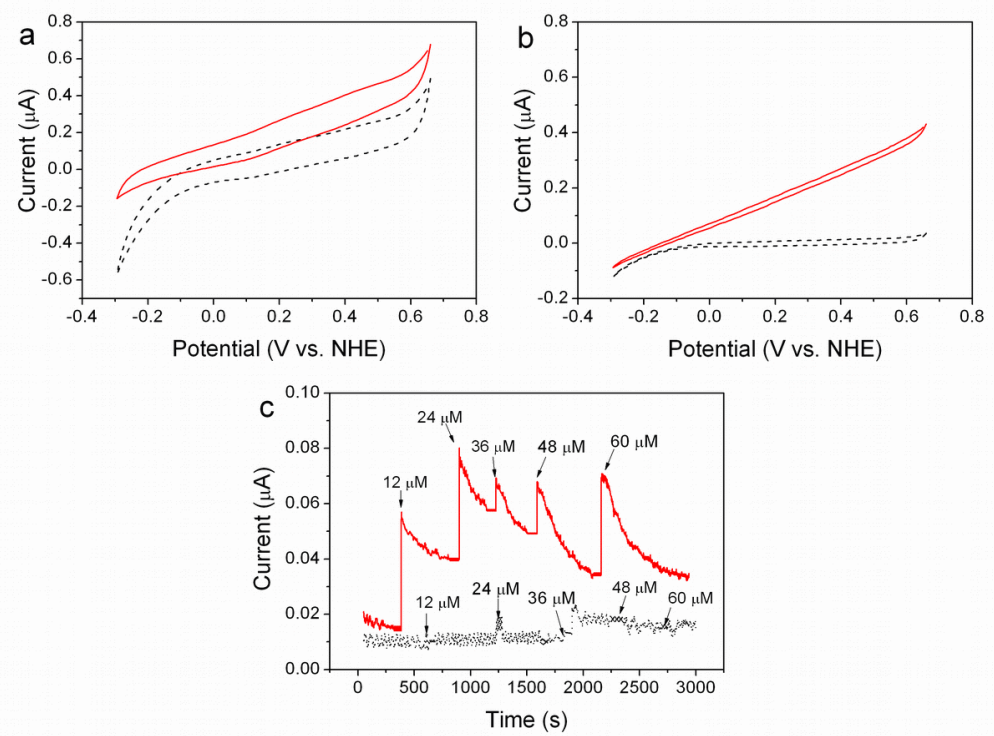

Figure 5. Electrocatalytic oxidation of NADH by complex I. CVs performed in $0.1 \mathrm{M}$ phosphate buffer, pH 7.0 in the absence (dashed line) and in the presence of $60 \mu \mathrm{M}$ NADH (solid line) with a Au/4ATP/PhL+C-I+DMN electrode: (a) CVs at $100 \mathrm{mV} / \mathrm{s}$; (b) $\mathrm{CVs}$ at $5 \mathrm{mV} / \mathrm{s}$. (c) Chronoamperommetry recorded in $0.1 \mathrm{M}$ phosphate buffer, $\mathrm{pH} 7.0$ with sequential additions of $\mathrm{NADH}$ (the concentration indicated in the graph is the final one in the solution) with $\mathrm{Au} / 4-\mathrm{ATP} / \mathrm{PhL}+\mathrm{C}-\mathrm{I}+\mathrm{DMN}$ (thick line) and $\mathrm{Au} / 4-\mathrm{ATP} / \mathrm{PhL}+\mathrm{DMN}$ (thin line) electrodes. 
Chronoamperometry experiments were also performed with the $\mathrm{Au} / 4-\mathrm{ATP} / \mathrm{PhL}+\mathrm{C}-\mathrm{I}+\mathrm{DMN}$ electrode poised at $+510 \mathrm{mV}$ (Fig. 5c). NADH aliquots were added sequentially up to a final concentration of $60 \mu \mathrm{M}$ to the quiescent solution. The oxidation current increased upon each addition of NADH, reaching a pseudo steady-state value after a few minutes. However, in the long term the steady-state current decreased even if the bulk NADH concentration was higher. This effect could be correlated to the diffusion of DMN, which plays the role of redox mediator in the electrocatalytic process, out of the biomimetic membrane towards the solution. As expected, the control experiment did without the enzyme $(\mathrm{Au} / 4-\mathrm{ATP} / \mathrm{PhL}+\mathrm{DMN})$ presents insignificant changes of the measured current upon NADH addition. This result confirms that the currents of NADH oxidation detected with the reconstituted complex I on membrane-modified electrodes are consequence of its enzymatic activity.

Proton translocation by the reconstituted complex I. In vivo, complex I couples NADH:quinone oxidoreduction to translocation of protons across the membrane. We have shown above that the reconstituted complex I on the modified gold electrodes was catalytically active for NADH:quinone oxidoreduction. In order to ascertain if the reconstituted enzyme was also able to translocate protons across the biomimetic membrane, we used the redox process of aniline dimers formed from the 4-ATP monolayer on the electrode surface as probe of local $\mathrm{pH}$ changes at the membrane/electrode interface. This aniline/quinone imine conversion involves $2 \mathrm{e}^{-} / 2 \mathrm{H}^{+}$, thus its formal redox potential should shift theoretically $-56 \mathrm{mV} / \mathrm{pH}$ unit at $25^{\circ} \mathrm{C} .{ }^{40}$ Therefore, to detect proton transport in the modified electrodes we measured the change of the oxidation peak potential of this process by differential pulse voltammetry (DPV), which is a more sensitive electrochemical technique for measuring peak shifts than cyclic voltammetry. First, we calibrated the system with a Au/4-ATP electrode, previously scanned several times between -0.1 and $+0.7 \mathrm{~V}$ vs. $\mathrm{Ag} / \mathrm{AgCl}$ in order to oxidize some of the 4-ATP groups on the surface to quinone diimines. The peak shifts of the DPV wave were measured in buffer solutions of different $\mathrm{pH}$ values. In this case the $\mathrm{pH}$ at the electrode/solution interface is the same as the bulk solution 
one because there is no supported membrane and the solution was stirred before the measurement. Fig. 6a shows that the peak potential shifted $-56 \mathrm{mV} / \mathrm{pH}$ unit, in agreement with theory.
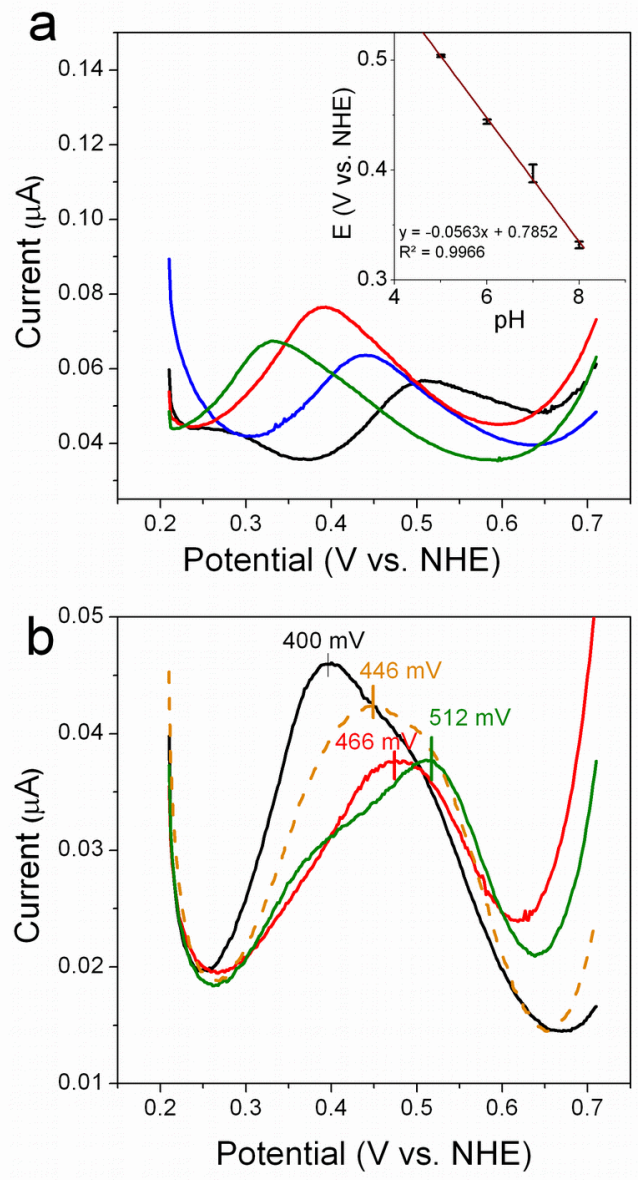

Figure 6. Electrochemical measurement of the $\mathrm{pH}$ at the electrode/membrane interface. (a) DPVs of a gold wire modified with a partially oxidized 4-ATP monolayer in $100 \mathrm{mM}$ phosphate buffer at different $\mathrm{pH}$ values. The inset shows the peak potential dependence on $\mathrm{pH}$ plot and the linear regression equation. (b) DPVs of $\mathrm{Au} / 4-\mathrm{ATP} / \mathrm{PhL}+\mathrm{C}-\mathrm{I}+\mathrm{DMN}$ in $1 \mathrm{mM}$ phosphate buffer, $\mathrm{pH}$ 7.0, $20 \mathrm{mM} \mathrm{Na}{ }_{2} \mathrm{~S}$ (red line); after addition of $60 \mu \mathrm{M}$ NADH (green line); after addition of $60 \mu \mathrm{M}$ NADH and 2,4-DNP $70 \mu \mathrm{M}$ (orange dashed line). The black line corresponds to the DPV of Au/4-ATP/PhL+DMN in presence of $60 \mu \mathrm{M}$ NADH. 
Fig. 6b shows typical DPV measurements obtained with a Au/4-ATP/PhL+C-I+DMN under $1 \mathrm{mM}$ buffer concentration conditions, in order to differentiate the $\mathrm{pH}$ value at the electrode/membrane interface from the bulk solution value (Fig. 6b, red line). Upon addition of $60 \mu \mathrm{M}$ NADH the peak potential shifted $46 \mathrm{mV}$ to higher potentials (Fig. 6b, green line), indicating that the local $\mathrm{pH}$ at the interface decreased almost one unit. The $\mathrm{pH}$ in the bulk solution, measured with a $\mathrm{pH}$-meter, did not change, as expected taking into account its large volume $(35 \mathrm{~mL})$. To be sure that the increase of the proton concentration in the interface was due to proton translocation across the biomimetic membrane, an ionophore $(2,4$ dinitrophenol, DNP) was added to the solution. Under those conditions, the peak potential shifted $66 \mathrm{mV}$ to lower potentials (Fig. 6b, orange dashed line), indicating an increase of the interface $\mathrm{pH}$. The shifts of the peak potential measurements were quite reproducible using several modified electrodes. The average shift was $+50 \pm 10 \mathrm{mV}$ (6 different electrodes) upon $60 \mu \mathrm{M}$ NADH addition.

Fig. $6 \mathrm{~b}$ also shows a control measurement done with a $\mathrm{Au} / 4-\mathrm{ATP} / \mathrm{PhL}+\mathrm{DMN}$, in which the enzyme was not present in the phopholipid bilayer (Fig. 6b, black line). In this case the addition of NADH did not change the peak potential, confirming that the $\mathrm{pH}$ changes at the membrane/electrode interface described above were due to the presence of complex I.

\section{Discussion}

In this work we present the first entire and functional reconstitution of a respiratory complex I on a biomimetic bilayer. Usually the studies of this enzyme are done with the two different domains separated and not in similar conditions to the natural ones. ${ }^{3,13,41}$. The AFM study shows that a phospholipid bilayer is formed on the Au (1111) surface modified with 4-ATP (the formation of a positively charged monolayer on the electrode surface favours the formation of a negatively charged phospholipid bilayer) ${ }^{23}$ and that hydrophilic domains of the complex I protrude out of the biomimetic membrane towards the solution. We measure by AFM a 6-8 nm height for this hydrophilic region. X-ray crystallography data indicate that the peripheral hydrophilic arm of the L-shaped complex I extends about $13 \mathrm{~nm}$ over the phospholipid bilayer. ${ }^{4}$ The difference between the AFM measuments and X-ray data could be due to the fact that the arm is not 
sitting at a right angle with respect to the membrane surface, as was determined from the X-ray data, and/or also to a possible flexibility of the domain that allows for a certain deformation upon contact with the AFM tapping tip while imaging. We can not assure that all enzyme molecules have the same orientation, but we can affirm there is a population that occupies $4-10 \%$ of the membrane surface whose conformation corresponds to the one shown in the scheme of Fig. 1. Although it is likely that in the initial proteo-lipid vesicles the complex I molecules are anchored on both sides, incorporation of the enzyme into the surface supported bilayers probably favours orienting the hydrophilic arm away from the gold surface. Very asymmetric proteins incorporated into supported lipid membranes in the presence of detergents are known to incorporate preferentially with one orientation. ${ }^{42}$ The impedance study confirmed the formation of the phospholipid bilayer over the gold wires used for the electrochemical measurements. ${ }^{43}$ The large increase on the charge resistance at the electrode surface measured upon overnight incubation in the liposome suspension is incompatible with just an adsorption of liposome vesicles, ${ }^{43}$ whereas it is in agreement with a phospholipid bilayer formation as observed by AFM. It is interesting to note that the impedance measurements indicate that the bilayer is more insulating, thus more compact, when it incorporates complex I. This suggests that the insertion of the hydrophobic domain of this enzyme in the biomimetic membrane stabilizes it, decreasing the number of defects in its structure. Nevertheless, higher values of charge resistance have been reported for other supported lipid layers, such as tethered bilayer membranes, which indicate that our biomimetic membrane is not defect free..$^{39,43,44}$

The advantage of reconstituting complex I on modified gold electrodes is that a panoply of electrochemical techniques can be used to study its functional properties. We show that not only the electrocatalytical oxidation of NADH can be studied, but also the coupled proton translocation across the membrane can be explored. The construction is functional only when the enzyme, the NADH and the quinone are present. The electrocatalytic currents measured are low, which are in agreement with the low enzyme coverage observed by AFM and with the low turnover of complex I from the thermophilic organism R. marinus for oxidizing NADH using DMN as electron acceptor (at $25^{\circ} \mathrm{C}$ ). ${ }^{18}$ The CV measured in presence of NADH do not show the typical electrocatalytic effect in which a plateau current is reached 
when the process is limited by mass transport of the substrate or enzymatic turnover. ${ }^{19}$ In fact, the CVs have similar shape (the catalytic current increases linearly with the overpotential) to those reported for the electrocatalytic behavior of other metalloenzymes, such as hydrogenases ${ }^{45}$ or the hydrophilic subunit of complex I. ${ }^{13}$ In these cases the CV shape was explained by the overlap of the electrocatalytic response of enzyme molecules with different orientations at the electrode surface. ${ }^{13,45}$ However, in these cases electrocatalysis was obtained by direct electron transfer of the enzyme, which was rate-limiting the process, whereas in our case we have a mediated process. This suggests that in our case the rate-limiting step is the re-oxidation of the quinone mediator (DMN) at the electrode. The control measurements of the work indeed indicate that the DMN electrochemistry is quite sluggish. Moreover, several works show that the electrochemistry of quinones becomes less reversible at lower $\mathrm{pH}$ and their oxidation waves shift to higher potentials. A possible explanation for the linear increase of the electrocatalytic currents observed in the $\mathrm{CV}$ of Fig $5 \mathrm{~b}$ is that the $\mathrm{pH}$ increase at the electrode/membrane interface, due to the proton translocation coupled to the enzymatic NADH oxidation, shifts the oxidation potential of DMN during the electrocatalytic measurement. This effect would be equivalent to the presence of redox mediator molecules with a wide range of potentials, thus leading to an electrocatalytic wave that does not reach a plateau. ${ }^{46}$

The generation of a proton-dependent redox process at the electrode surface, which is not involved in the enzymatic process, allows monitoring quantitatively the changes of $\mathrm{pH}$ at the membrane/electrode interface. Therefore, a fast and simple electrochemical method is developed for studying proton translocation across supported membranes that does not require the use of the normally used fluorescence probes. $^{6,710-12}$ However, it must be taken into account that the measured local $\mathrm{pH}$ is the net balance between the proton translocation from the bulk solution to the electrode/membrane interface by complex I and the proton diffusion in the opposite direction through the supported membrane defects. In a previous work we showed that such type of supported phospholipid bilayer was in part permeable to positively charged compounds through the defects. ${ }^{23}$ 


\section{Conclusions}

This work presents a new methodological approach to study respiratory complex I. The employed system allows reconstituting this membrane enzyme in a configuration very similar to its natural one, and taking advantage of the high sensitivity, versatility and reliability of electrochemical methods for studying complex I functional properties. Both electron transfer and proton translocation by respiratory complex I were monitored on the electrode, showing electrocatalytic oxidation of NADH mediated by the quinone pool within the biomimetic membrane and proton accumulation at the membrane/electrode interface.

Furthermore, this approach opens the way for developing new assays for screening drug effects on mitochondrial function. ${ }^{29}$

\section{Supporting Information}

Additional AFM images, impedance data and CVs of modified gold electrodes. This material is available free of charge via the Internet at http://pubs.acs.org.

\section{Corresponding author}

Telephone: +34915854802. Fax:+34915854760. E-mail: alopez@icp.csic.es

\section{Acknowledgements}

This work was funded by the Spanish MINECO (project CTQ2012-32448) and by Fundação para a Ciência e a Tecnologia (PTDC/BBB-BQB/2294/2012 to M.M.P.). The work was also supported by Fundação para a Ciência e a Tecnologia through grant \# PEst-OE/EQB/LA0004/2011. M.P. and O.G.-S. acknowledge the Ramon y Cajal and the FPI programs respectively from the Spanish MINECO. A.P.B. is recipient of a grant from Fundação para a Ciência e a Tecnologia (SFRH/BPD/80741/2011).

\section{References}


1 Schapira, A. H. V. Human complex I defects in neurodegenerative diseases. Biochim. Biophys. Acta 1998, 1364, 261-270.

2 Hunte, C.; Zickermann, V.; Brandt, U. Functional modules and structural basis of conformational coupling in mitochondrial complex I. Science 2010, 329, 448-451.

3 Sazanov, L. A.; Hinchliffe, P. Structure of the hydrophilic domain of respiratory complex I from Thermus thermophilus. Science 2006, 311, 1430-1436.

4 Efremov, R. G.; Baradaran, R.; Sazanov, L.A. The architecture of respiratory complex I. Nature 2010, 465, 441-446.

5 Baradaran, R.; Berrisford, J.M.; Minhas, G.S.; Sazanov, L.A. Crystal structure of the entire respiratory complex I. Nature 2013, 494, 443-448.

6 Bogachev, A. V.; Murtazina, R. A.; Skulachev, V. P. $\mathrm{H}^{+} / \mathrm{e}^{-}$stoichiometry for NADH dehydrogenase I and dimethyl sulfoxide reductase in anaerobically grown Escherichia coli cells. $J$. Bacteriol. 1996, 178, 6233-6237.

7 Galkin, A. S.; Grivennikova, V. G.; Vinogradov, A. D. $\mathrm{H}^{+} / 2 \mathrm{e}^{-}$stoichiometry in NADH-quinone reductase reactions catalyzed by bovine heart submitochondrial particles. FEBS Lett. 1999, 451, 157-161.

8 Steuber, J.; Schmid, C.; Rufibach, M.; Dimroth, P. $\mathrm{Na}^{+}$translocation by complex I (NADH:quinone oxidoreductase) of Escherichia coli. Mol. Microbiol. 2000, 35, 428-434.

9 Gemperli, A. C.; Dimroth, P.; Steuber, J. The respiratory complex I (NDH I) from Klebsiella pneumoniae, a sodium pump. J. Biol. Chem. 2002, 277, 33811-33817.

10 Batista, A. P.; Fernandes, A.; Louro, R. O.; Steuber, J.; Pereira, M. M. Energy conservation by Rhodothermus marinus respiratory complex I. Biochim. Biophys. Acta 2010, 1797, 509-515.

11 Batista, A. P.; Pereira, M. M. Sodium influence on energy transduction by complexes I from Escherichia coli and Paracoccus denitrificans. Biochim. Biophys. Acta 2010, 1807, 286-292.

12 Stolpe, S.; Friedrich, T. The Escherichia coli NADH:ubiquinone oxidoreductase (complex I) is a primary proton pump but may be capable of secondary sodium antiport. J. Biol. Chem. 2004, 279, 18377-18383. 
13 Barker, C. D.; Reda, T.; Hirst, J. The flavoprotein subcomplex of complex I (NADH:ubiquinone oxidoreductase) from bovine heart mitochondria: insights into the mechanisms of $\mathrm{NADH}$ oxidation and $\mathrm{NAD}^{+}$reduction from protein film voltammetry. Biochemistry 2007, 46, 3454-3464.

14 Marshall, D.; Rich, P. R. Studies of complex I by fourier transform infrared spectroscopy. Methods Enzymol. 2009, 456, 53-74.

15 Bridges, H. R.; Bill, E.; Hirst J. Mösbauer spectroscopy on respiratory complex I: the ironsulfur cluster ensemble in the NADH-reduced enzyme is partially oxidized. Biochemistry 2012, 51, 149158.

16 Hielscher, R.; Yegres, M.; Voicescu, M.; Gnandt, E.; Friedrich, T.; Hellwig, P. Characterization of two quinone radicals in the NADH:ubiquinone oxidoreductase from Escherichia coli by a combined fluorescence spectroscopic and electrochemical approach. Biochemistry 2013, 52, 8993-9000.

17 Yano, T.; Dunham, W. R.; Ohnishi, T. Characterization of the $\Delta \mu_{\mathrm{H}}{ }^{+}$-sensitive ubisemiquinone species $\left(\mathrm{SQ}_{\mathrm{Nf}}\right)$ and the interaction with cluster N2: new insight into the energy-coupled electron transfer in complex I. Biochemistry 2005, 44, 1744-1754.

18 Batista, A. P.; Marreiros, B. C.; Pereira, M. M. Decoupling of the catalytic and transport activities of complex I from Rhodothermus marinus by sodium/proton antiporter inhibitor. ACS Chem. Biol. 2011, 6, 477-483.

19 Leger, C.; Bertrand, P. Direct electrochemistry of redox enzymes as a tool for mechanistic studies. Chem. Rev. 2008, 28, 2379-2438.

20 Jeuken, L. J. C. Electrodes for integral membrane systems. Nat. Prod. Rep. 2009, 26, 12341240.

21 Lipkowski, J. Building biomimetic membrane at a gold electrode surface. Phys. Chem. Chem. Phys. 2010, 12,13874-13887.

22 Marchal D.; Pantigny, J.; Laval, J. M.; Moiroux, J.; Bourdillon, C. Rate constants in two dimensions of electron transfer between pyruvate oxidase a membrane enzyme, and ubiquinone (coenzyme Q8), its water-insoluble electron carrier. Biochemistry 2001, 40, 1248-1256. 
23 Gutiérrez-Sanchez, C.; Olea, D.; Marques, M.; Fernández, V. M.; Pereira, I. A. C.; Vélez, M.; De Lacey, A. L. Oriented immobilization of a membrane-bound hydrogenase onto an electrode for direct electron transfer. Langmuir 2011, 27, 6449-6457.

24 McMillan, D. G. G.; Marrit, S. J.; Butt, J. N.; Jeuken, L. J. C. Menaquinone-7 is specific cofactor in tetraheme quinol dehydrogenase CymA. J. Biol. Chem 2012, 287, 14215-14225.

25 Ataka, K.; Giess, F.; Knoll, W.; Naumann, R.; Haber-Pohlmeier, S.; Richter, B.; Heberle, J. Oriented attachment and membrane reconstitution of his-tagged cytochrome $c$ oxidase to a gold electrode: In situ monitoring by surface-enhanced infrared absortion spectroscopy. J. Am. Chem. Soc. 2004, 126, 16199-16206.

26 Ciaccafava, A.; Infossi, P.; Ilbert, M.; Guiral, M.; Lecomte, S.; Giudici-Orticoni, M. T.; Lojou, E. Electrochemistry, AFM, and PM-IRRA Spectroscopy of Immobilized Hydrogenase: Role of a Hydrophobic Helix in Enzyme Orientation for Efficient $\mathrm{H}_{2}$ Oxidation. Angew. Chem.-Int. Ed. 2012, 51, 953-956.

27 Kozuch, J.; Steinem, C.; Hildebrandt, P.; Millo, D. Combined electrochemistry and surfaceenhanced infrared absorption spectroscopy of gramacidin A incorporated into tethered bilayer lipid membranes. Angew. Chem.-Int. Ed. 2012, 51, 8114-8117.

28 Melin, F.; Hellwig, P. Recent advances in the electrochemistry and spectroelectrochemistry of membrane proteins. Biol. Chem. 2013, 394, 593-609.

29 Preissl, S.; Bick, I.; Obrdlik, P.; Diekert, K.; Gul, S.; Gribbon, P. Development of an assay for complex I/complex III of the respiratory chain using solid supported membranes and its application in mitochondrial toxicity screening in drug discovery. Assay Drug Develop. Technol. 2011, 9, 147-156.

30 Pereira, M. M.; Carita, J. N.; Teixeira, M. Membrane-bound electron transfer chain of the thermohalophilic bacterium Rhodothermus marinus: a novel multihemic cytochrome $b c$, a new complex III. Biochemistry 1999, 38, 1268- 1275. 
31 Fernandes, A. S.; Sousa, F. L.; Teixeira, M.; Pereira, M. M. Electron paramagnetic resonance studies of the iron-sulfur centers from complex I of Rhodothermus marinus. Biochemistry 2006, 45, 1002-1008.

32 Kruber, O. On the 2,3-dimethyl-naphthaline in pit coal tar. Berichte Deutsche Chem. Gesellschaft 1929, 62, 3044-3047.

33 Randles, J. E. B. Kinetics of rapid electrode reactions. Discuss Faraday Soc. 1947, 1, 11-19.

34 Zoski, Z. G. Handbook of Electrochemistry pp 454-458, Elsevier, Amsterdam, 2007.

35 Blaedel, W. J.;Jenkins, R. A. Study of the electrochemical oxidation of reduced nicotinamide adenine dinucleotide. Anal. Chem. 1975, 47, 1337-1343.

36 Sánchez, S.; Arratia, A.; Córdova, R.; Gómez, H.; Schrebler, R. Electron transport in biological procesess II: electrochemical behaviour of $\mathrm{Q}_{10}$ immersed in a phospholipidic matrix added on a pyrolitic graphite electrode. Bioelectrochem. Bioenerg. 1995, 36, 67-71.

37 Marchal, D.; Boireau, W. ; Laval, J. M.; Bourdillon, C.; Moiroux, J. Kinetics of redox conversion at a gold electrode of water-insoluble ubiquinone $\left(\mathrm{UQ}_{(10)}\right)$ and plastoquinone $\left(\mathrm{PQ}_{(9)}\right)$ incorporated in supported phospholipid layers. J. Electroanal. Chem. 1998, 451, 139-144.

38 Gordillo, G. J.; Schiffrin, D. J. The electrochemistry of ubiquinone-10 in a phospholipid model membrane.Faraday Discuss. 2000, 116, 89-107.

39 Martensson, C. ; Agmo Hernandez, V. Ubiquinone-10 in gold-immobilized lipid membrane structures acts as a sensor for acetylcholine and other tetraalkylammonium cations. Bioelectrochemistry 2012, 88, 171-180.

40 Raj, C. R.; Kitamura, F.; Ohsaka T. Electrochemical and in situ FTIR spectroscopic investigation on the electrochemical transformation of 4-aminothiophenol on a gold electrode in neutral solution. Langmuir 2001, 17, 7378-7386.

41 Hellwig, P.; Scheide, D.; Bungert, S.; Mäntele, W.; Friedrich, T. FT-IR spectroscopic characterization of NADH:ubiquinone oxidoreductase (complex1) from Escherichia coli: oxidation of FeS 
cluster N2 is coupled with the protonation of an aspartate or glutamate side chain Biochemistry 2000, 39, 10884-10891.

42 Milhiet, P.; Gubellini, F.; Berquand, A.; Dosset, P.; Rigaud, J. L.; Le Grimellec, C.; Lévy, D. High-resolution AFM of membrane proteins directly incorporated at high density in planar lipid bilayer. Biophys. J. 2006, 91, 3268-3275.

43 Jeuken, L. J. C.; Connell, S. D.; Nurnabi, M.; O'Reilly, J.; Henderson, P. J. F.; Evans, D. H.; Bushby, R. J. Direct electrochemical interaction between a modified gold electrode and a bacterial membrane extract. Langmuir 2005, 21, 1481-1488.

44 Jeuken, L. J. C.; Bushby, R. J.; Evans, S. D. Proton transport into a tethered bilayer lipid membrane. Electrochem. Commun.2007, 9, 610-614.

45 Leger, C.; Jones, A. K.; Albracht, S. P. J.; Armstrong, F. A. Effect of a dispersion of interfacial electron transfer rates on steady state catalytic electron transport in [NiFe]-hydrogenase and other enzymes. J. Phys. Chem. B 2002, 106, 13058-13063.

46 Jiang, R.; Anson, F. C. The origin of inclined plateau currents in steady-state voltammograms for electrode processes involving electrocatalysis, J. Electroanal. Chem. 1991, 305, 171-184. 


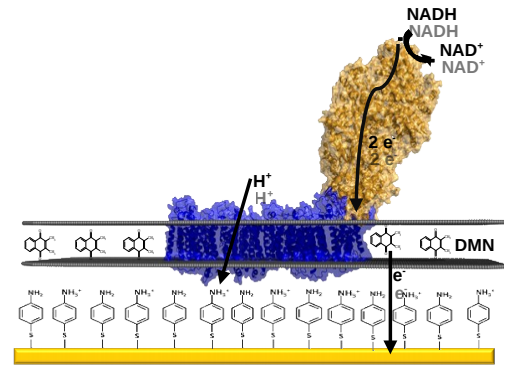

